\title{
Stringy corrections to the entropy of electrically charged supersymmetric black holes with $\mathrm{AdS}_{5} \times \mathrm{S}^{\mathbf{5}}$ asymptotics
}

\author{
João F. Melo ${ }^{1, *}$ and Jorge E. Santos ${ }^{1,2, \dagger}$ \\ ${ }^{1}$ DAMTP, Centre for Mathematical Sciences, University of Cambridge, \\ Wilberforce Road, Cambridge CB3 OWA, United Kingdom \\ ${ }^{2}$ Institute for Advanced Study, Princeton, New Jersey 08540, USA
}

(Received 18 August 2020; accepted 8 February 2021; published 16 March 2021)

\begin{abstract}
We study the leading $\alpha^{\prime}$ corrections to the entropy of certain black holes with $\mathrm{AdS}_{5} \times S^{5}$ asymptotics. We find that, in the supersymmetric limit, the entropy does not receive $\alpha^{\prime}$ corrections. This result strengthens recent calculations that match the index of $\mathcal{N}=4$ super-Yang-Mills with the corresponding partition function in the supersymmetric limit. In the small temperature regime, we find that the entropy corrections are concordant with the weak gravity conjecture.
\end{abstract}

DOI: 10.1103/PhysRevD.103.066008

\section{INTRODUCTION}

Quantum gravity remains a largely unexplored frontier. However, due to the seminal work in black hole thermodynamics in the seventies [1-9], we know that, whatever the ultimate unifying theory is, it should reproduce the Hawking effect and give a microscopic derivation of the Bekenstein-Hawking black hole entropy in the appropriate semiclassical limit. To date, string theory appears to be the only candidate for a quantum theory of gravity that explains both of these effects in an ambiguity free manner at a microscopic level [10-13]. In particular, the seminal work of [10] provided a beautiful matching between the Bekenstein-Hawking entropy of certain five-dimensional supersymmetric black holes with asymptotically flat boundary conditions and the counting of specific supersymmetric states. Since then, a number of generalizations of this work have been accomplished for black holes with more complex topologies (see, e.g., [14]).

However, this matching has only been accomplished for black holes with asymptotically flat boundary conditions. One might wonder how to extend these results to asymptotically anti-de Sitter (AdS) spacetimes, for which we have the so-called AdS/CFT correspondence [15-18]. In its original form, the AdS/CFT correspondence relates four-dimensional $\mathcal{N}=4$ super-Yang-Mills (SYM) with gauge group $S U(N)$ and 't Hooft coupling $\lambda$, to type IIB

\footnotetext{
jifm54@cam.ac.uk

jss55@cam.ac.uk
}

Published by the American Physical Society under the terms of the Creative Commons Attribution 4.0 International license. Further distribution of this work must maintain attribution to the author(s) and the published article's title, journal citation, and DOI. Funded by SCOAP ${ }^{3}$. superstring theory with string coupling $g_{s}$, string length $\ell_{s} \equiv \sqrt{\alpha^{\prime}}$ on $\mathrm{AdS}_{5} \times S^{5}$ with radius $L$ and $N$ units of $F_{(5)}$ flux through the $S^{5}$. The field theory is thought to live at the conformal boundary of $\mathrm{AdS}_{5}$, and for this reason the correspondence is said to be holographic in nature. The string theory side is often referred to as the "bulk" and the field theory side as the "boundary".

The parameters on each side of the AdS/CFT correspondence are related via

$$
\frac{\lambda}{N}=2 \pi g_{s} \quad \text { and } \quad 2 \lambda=\frac{L^{4}}{\ell_{s}^{4}} .
$$

However, it remains a challenge to understand string theory for generic values of $g_{s}$, so one usually takes $N \rightarrow+\infty$, at fixed $\lambda$, so that $g_{s} \rightarrow 0$. Under these assumptions, the bulk theory reduces to a classical theory of strings. To simplify matters further, we can also take $\lambda$ to be large, but not necessarily infinite. On the field theory side, we are thus looking at strong coupling effects, and on the gravity side we have a supergravity theory. Corrections to the strict $\lambda \rightarrow+\infty$ limit appear in the bulk as higher derivative terms which account for finite size string corrections.

The problem of reproducing the entropy of certain black hole solutions in global $\mathrm{AdS}_{5}$ on the string theory side is now mapped into a counting problem of certain states on the field theory. Because we are interested in global $\mathrm{AdS}_{5}$, the field theory is thought to live on $\mathbb{R}_{t} \times S^{3}$. The holographic description of electrically charged supersymmetric black holes with $\operatorname{AdS} \times S^{5}$ asymptotics is in terms of states of the dual $\mathcal{N}=4 \mathrm{SYM}$ that preserve only one of the available 16 supercharges. Such states should be counted (with sign) by the superconformal index. However, early attempts to compute this index gave an order one 
result [19], whereas the entropy of $\mathrm{AdS}_{5}$ black holes scales with $N^{2}$. It was not until recently that this long-standing problem was partially solved. In particular, [20-36] have argued that, upon using complex chemical potentials, the cancellations between fermionic and bosonic degrees of freedom observed in [19] can be avoided. This leads to an index of order $N^{2}$, whose associated entropy matches those of known supersymmetric black holes [37-39]. This body of work thus provides overwhelming evidence that whether we compute the entropy via the index or via a more standard calculation using the partition function of $\mathcal{N}=4$ SYM, the results should agree with each other. It should be noted that this latter quantity can only be computed via an indirect bulk calculation using the Bekenstein-Hawking entropy.

The matching between the partition function calculation and index leads to a number of fascinating predictions. In particular, since the index cannot exhibit a dependence on continuous parameters (except perhaps when wall crossing is observed, see, e.g., [40,41]), we expect the counting on the field theory side to not depend on the 't Hooft coupling $\lambda$. On the bulk side of the story, because we are computing directly a partition function, this is not an obvious fact given we know that the classical equations of motion of type IIB supergravity do admit corrections in $\alpha^{\prime}$, due to finite size stringy effects. These, in the small $\alpha^{\prime}$ limit, appear as higher-derivative corrections to the equations of motion of type IIB supergravity. The first nontrivial corrections for supergravity configurations that only involve the metric $g$ and five-form $F_{(5)}$ were worked out in $[42,43]$, following the seminal results of [44].

\section{THE BLACK HOLES}

We focus on black hole solutions of five-dimensional minimal gauged supergravity, whose action comprises a fivedimensional metric $g$ and a field strength $F=\mathrm{d} A$ and reads

$$
\begin{aligned}
S_{5 D}= & \frac{1}{16 \pi G_{5}} \int_{\mathcal{M}} \mathrm{d}^{5} x \sqrt{-g}\left(R+\frac{12}{L^{2}}-\frac{1}{4} F_{a b} F^{a b}\right. \\
& \left.+\frac{1}{12 \sqrt{3}} \varepsilon^{a b c d e} F_{a b} F_{c d} A_{e}\right) .
\end{aligned}
$$

Known black hole solutions in this theory carry one electric charge $Q$ and two angular momenta $J_{1}, J_{2}$. For simplicity, we focus on the case where $J_{1}=J_{2}=J$. The equations of motion derived from Eq. (2) read

$$
\begin{aligned}
R_{a b}-\frac{g_{a b}}{2} R-\frac{6}{L^{2}} g_{a b} & =\frac{1}{2}\left(F_{a}{ }^{c} F_{b c}-\frac{g_{a b}}{4} F^{c d} F_{c d}\right), \\
\nabla_{a} F^{a b} & =\frac{1}{4 \sqrt{3}} \varepsilon^{\mathrm{bcdef}} F_{c d} F_{e f} .
\end{aligned}
$$

We are interested in the $\alpha^{\prime}$ corrections to the entropy of the black holes constructed in [45], which read

$$
\begin{aligned}
\mathrm{d} s_{5 D}^{2} & =-\frac{f}{h} \mathrm{~d} t^{2}+\frac{\mathrm{d} r^{2}}{f}+\frac{r^{2}}{4}\left(\sigma_{1}^{2}+\sigma_{2}^{2}\right)+\frac{r^{2}}{4} h\left(\sigma_{3}-W \mathrm{~d} t\right)^{2} \\
A & =\frac{\sqrt{3} \tilde{Q}}{r^{2}}\left(\mathrm{~d} t-\frac{\tilde{J}}{2} \sigma_{3}\right)
\end{aligned}
$$

where $\sigma_{1}, \sigma_{2}, \sigma_{3}$ are the usual left-invariant one-forms of $S^{3}$,

$$
\begin{aligned}
& \sigma_{1}=-\sin \psi \mathrm{d} \theta+\cos \psi \sin \theta \mathrm{d} \phi, \\
& \sigma_{2}=\cos \psi \mathrm{d} \theta+\sin \psi \sin \theta \mathrm{d} \phi, \\
& \sigma_{3}=\mathrm{d} \psi+\cos \theta \mathrm{d} \phi,
\end{aligned}
$$

and

$$
f=\frac{r^{2}}{L^{2}}+1-\frac{2 \tilde{M}}{r^{2}}(1-\chi)+\frac{\tilde{Q}^{2}}{r^{4}}\left(1-\frac{\tilde{J}^{2}}{L^{2}}+\frac{2 \tilde{M} L^{2} \chi}{\tilde{Q}^{2}}\right),
$$

$W=\frac{2 \tilde{J}}{r^{2} h}\left(\frac{2 \tilde{M}+\tilde{Q}}{r^{2}}-\frac{\tilde{Q}^{2}}{r^{4}}\right)$,

$h=1-\frac{\tilde{J}^{2} \tilde{Q}^{2}}{r^{6}}+\frac{2 \tilde{J}^{2}(\tilde{M}+\tilde{Q})}{r^{4}}$,

where $L^{2} \chi \equiv \tilde{J}^{2}(1+\tilde{Q} / \tilde{M})$. The constants $\tilde{M}$, $\tilde{Q}$, and $\tilde{J}$ parametrize the energy $M$, electric charge $Q$, and angular momentum $Q$ as

$$
\begin{aligned}
M & =\frac{3 \tilde{M} \pi}{4 G_{5}}\left(1+\frac{\chi}{3}\right), \\
J & =\frac{\tilde{J} \pi}{4 G_{5}}(2 \tilde{M}+\tilde{Q}), \\
Q & =\frac{\sqrt{3} L \pi \tilde{Q}}{4 G_{5}}
\end{aligned}
$$

The black hole event horizon is the null hypersurface $r=r_{+}$, with $r_{+}$being the largest real positive root of $f(r)$. The associated Hawking temperature $T$, entropy $S$, chemical potential $\mu$, and angular velocity $\Omega$ can be found in [45]. It is then a simple exercise to check that all thermodynamic quantities satisfy the first law of black hole mechanics,

$$
\mathrm{d} E=T \mathrm{~d} S+\mu \mathrm{d} Q+\Omega \mathrm{d} J .
$$

The Gibbs free energy is then constructed in the usual manner via $G=E-T S-\mu Q-\Omega J$. One can show that $G / T$ agrees with the Euclidean on-shell action (2) up to the 
usual Gibbons-Hawking-York [46,47] term and boundary counterterms $[48,49]$.

Finally, with our normalizations for $F$, the BPS condition is given by [50]

$$
\Delta \equiv M-\frac{2}{L} J-\frac{\sqrt{3}}{L} Q \geq 0 .
$$

The saturation of the BPS condition occurs only for supersymmetric solutions. Similar BPS bound has been shown not to receive $\alpha^{\prime}$ corrections even for asymptotically flat black holes [51]. The AdS BPS condition (9), together with the first law, implies $T=0, \Omega=2 / L$ and $\mu=\sqrt{3} / L$, which in turn yield

$$
\begin{aligned}
& \tilde{Q}=\tilde{Q}_{\mathrm{BPS}} \equiv r_{+}^{2}\left(1+\frac{r_{+}^{2}}{2 L^{2}}\right), \\
& \tilde{J}=\tilde{J}_{\mathrm{BPS}} \equiv \frac{L r_{+}^{2}}{r_{+}^{2}+2 L^{2}} .
\end{aligned}
$$

Note that even though the solutions (4) appear to depend on three parameters $(\tilde{M}, \tilde{Q}, \tilde{J})$, the BPS condition reduces this family to a one-parameter family, despite the fact that extremal black holes form a two-parameter family of solutions. We remark that $[52,53]$ provided strong numerical evidence for the existence of a new two-parameter family of supersymmetric black holes, whose role in this story remains to be understood. One can also show that demanding the absence of naked singularities in (4a) implies that $L>\tilde{J}$ [54].

Since the $\alpha^{\prime}$ corrections are only known in type IIB supergravity, we uplift the solutions (4) to ten dimensions. Using the results of [55-57], one can show that Eq. (4) oxidizes to the following solution of type IIB supergravity:

$$
\begin{gathered}
\mathrm{d} s^{2}=\mathrm{d} s_{5 D}^{2}+L^{2}\left[\left(\mathrm{~d} \Psi+\mathbb{A}-\frac{A}{\sqrt{3} L}\right)^{2}+\mathrm{d} \mathbb{C P} \mathbb{P}^{2}\right], \\
G_{(5)}=\frac{r^{3}}{2 L} \mathrm{~d} t \wedge \mathrm{d} r \wedge \sigma_{1} \wedge \sigma_{2} \wedge \sigma_{3}+\frac{L^{3}}{2 \sqrt{3}} \rrbracket \wedge \star_{5} F, \\
F_{(5)}=G_{(5)}+\star_{10} G_{(5)}
\end{gathered}
$$

where $\star_{5}$ is the five-dimensional Hodge dual obtained using the line element (4a), $\star_{10}$ is the Hodge dual obtained using the ten-dimensional line element $(11 \mathrm{a}), \mathrm{dCP} \mathbb{P}^{2}$ is the standard Fubini-Study metric on $\mathbb{C P}^{2}$ and $\mathbb{J}=\mathrm{d} \mathbb{A}$ is its associated Kähler form.

\section{EVALUATING THE CORRECTIONS}

The action [58] with the leading order $\alpha^{\prime}$ correction

\begin{tabular}{|c|c|}
\hline$n_{i}$ & $M_{i}$ \\
\hline-43008 & $C_{a b c d} C_{a b e f} C_{c e g h} C_{d g f h}$ \\
\hline 86016 & $C_{a b c d} C_{a e c f} C_{b g e h} C_{d g f h}$ \\
\hline 129024 & $C_{a b c d} C_{a e f g} C_{b f h i} \mathcal{T}_{\text {cdeghi }}$ \\
\hline 30240 & $C_{a b c d} C_{a b c e} \mathcal{T}_{\text {dfghij }} \mathcal{T}_{\text {efhgij }}$ \\
\hline 7392 & $C_{a b c d} C_{a b e f} \mathcal{T}_{\text {cdghij }} \mathcal{T}_{\text {efghij }}$ \\
\hline-4032 & $C_{a b c d} C_{a e c f} \mathcal{T}_{\text {beghij }} \mathcal{T}_{\text {dfghij }}$ \\
\hline-4032 & $C_{a b c d} C_{a e c f} \mathcal{T}_{\text {bghdij }} \mathcal{T}_{\text {eghfij }}$ \\
\hline-118272 & $C_{a b c d} C_{a e f g} \mathcal{T}_{\text {bcehij }} \mathcal{T}_{\text {dfhgij }}$ \\
\hline-26880 & $C_{a b c d} C_{a e f g} \mathcal{T}_{\text {bcehij }} \mathcal{T}_{\text {dhifgj }}$ \\
\hline 112896 & $C_{a b c d} C_{a e f g} \mathcal{T}_{\text {bcfhij }} \mathcal{T}_{\text {dehgij }}$ \\
\hline-96768 & $C_{a b c d} C_{a e f g} \mathcal{T}_{\text {bcheij }} \mathcal{T}_{\text {dfhgij }}$ \\
\hline 1344 & $C_{a b c d} \mathcal{T}_{\text {abefgh }} \mathcal{T}_{\text {cdeijk }} \mathcal{T}_{\text {fghijk }}$ \\
\hline-12096 & $C_{a b c d} \mathcal{T}_{\text {abefgh }} \mathcal{T}_{\text {cdfijk }} \mathcal{T}_{\text {eghijk }}$ \\
\hline-48384 & $C_{a b c d} \mathcal{T}_{\text {abefgh }} \mathcal{T}_{\text {cdfijk }} \mathcal{T}_{\text {egihjk }}$ \\
\hline 24192 & $C_{a b c d} \mathcal{T}_{\text {abefgh }} \mathcal{T}_{\text {cefijk }} \mathcal{T}_{\text {dghijk }}$ \\
\hline 2386 & $\mathcal{T}_{\text {abcdef }} \mathcal{T}_{\text {abcdgh }} \mathcal{T}_{\text {egijkl }} \mathcal{T}_{\text {fijhkl }}$ \\
\hline-3669 & $\mathcal{T}_{\text {abcdef }} \mathcal{T}_{\text {abcdgh }} \mathcal{T}_{\text {eijgkl }} \mathcal{T}_{\text {fikhjl }}$ \\
\hline-1296 & $\mathcal{T}_{\text {abcdef }} \mathcal{T}_{\text {abcghi }} \mathcal{T}_{\text {dejgkl }} \mathcal{T}_{\text {fhkijl }}$ \\
\hline 10368 & $\mathcal{T}_{\text {abcdef }} \mathcal{T}_{\text {abcghi }} \mathcal{T}_{\text {dgjekl }} \mathcal{T}_{\text {fhkijl }}$ \\
\hline 2688 & $\mathcal{T}_{\text {abcdef }} \mathcal{T}_{\text {abdegh }} \mathcal{T}_{\text {cgijkl }} \mathcal{T}_{\text {fjkhil }}$ \\
\hline
\end{tabular}
is [42]
TABLE I. Table detailing the $\alpha^{3}$ corrections of any solution in type IIB supergravity with nontrivial metric $g$ and five-form $F_{(5)}$. Following [42], all tensor monomials are written with all indices lower.

$S_{I I B}=\frac{1}{16 \pi G_{10}} \int_{\mathcal{M}_{10}} \mathrm{~d}^{10} x \sqrt{-g}\left(R-\frac{1}{4 \times 5 !} F_{(5)}^{2}+\gamma \mathcal{W}\right)$,

where $\mathcal{W}$ is given by

$$
\mathcal{W} \equiv \frac{1}{86016} \sum_{i=1}^{20} n_{i} M_{i}
$$

with all 20 monomials given in Table I and [59]

$$
\mathcal{T}_{\text {abcdef }}=i \nabla_{a} F_{\text {bcdef }}+\frac{1}{16}\left(F_{\mathrm{abcmn}} F_{\mathrm{def}}{ }^{m n}-3 F_{\mathrm{abfmn}} F_{\mathrm{dec}}{ }^{m n}\right) \text {. }
$$

Finally, we also have

$$
\gamma=\frac{\alpha^{\prime 3}}{16} \frac{\pi^{3}}{8} \zeta(3)
$$

We notice that Table I corrects some typos in the final table of [42].

Our objective is to use these results to compute the leading correction to the entropy of the black hole solution 
detailed in (4). Naively, one might think that we would need to solve the equations of motion from the action (12) and only then evaluate the correction to the entropy. However, due to the work in [60] (whose results straightforwardly generalize to the case at hand), one in fact only needs to know the 0th order solution, and evaluate that on the corrected action to get the leading corrections to the entropy.

This is a major simplification and is one of the main reasons this work is possible. However, it is still not a trivial task to evaluate all the monomials from Table I without accidentally inserting typos. Therefore, one of the key steps we had to take was validating our calculations. We wrote two pieces of code independently from one another, only comparing them at the end to make sure they agreed. We started by confirming the results of [42] to make sure there were no mistakes when copying the monomials from Table I.

Only after we had two matching codes that confirmed the results in [42] did we insert the solution (4). And even then, to be completely certain we had no typos or no convention compatibility issues, not only did we include many consistency checks throughout the code, e.g., confirming we indeed solved the correct equations of motion, but we used two different parametrizations. One of them using a $\mathbb{C P} \mathbb{P}^{2}$ fibration and another using a more direct method using the coordinates as originally written in [56]. The $\mathbb{C} \mathbb{P}^{2}$ fibration is the more efficient method and therefore is the one included in the Supplemental Material [61]. However, the direct method is more amenable to generalization for the case of different angular momenta [56,62], which we leave for future work.

After the colossal amount of dust settles, all 20 terms in Table I are nonvanishing on our solutions, and yet the final result appears simple, which gives further confidence in our answer. Using the relation between the Gibbs free energy $G$ and the Euclidean action obtained from (12), we find that the stringy correction to the Gibbs free energy at fixed chemical potential $\mu$, angular velocity $\Omega$, and temperature $T$ reads

$$
\begin{aligned}
(\delta G)_{\mu, \Omega, T}= & -\frac{12 \pi^{3} N^{2} \alpha^{\prime 3}(\tilde{M}+\tilde{Q})^{2} \zeta(3)}{L^{12} r_{+}^{15}\left(9 L^{2}-\tilde{J}^{2}\right)} \\
& \times\left(L^{2}-\tilde{J}^{2}\right)^{3} \Delta\left(\Delta+\frac{4}{L} J\right) \leq 0 .
\end{aligned}
$$

It is a simple matter to compute the variation in entropy, $(\delta S)_{Q, J, M}$, at fixed asymptotic charges $Q, J$, and $M$ from $(\delta G)_{\mu, \Omega, T}$. In particular, we can follow the same steps as in [60] to show that

$$
(\delta S)_{Q, J, M}=-T^{-1}(\delta G)_{\mu, \Omega, T}
$$

Equations (16) and (17) are the main result of this paper, whose physical significance we discuss next.

\section{INTERPRETATION OF RESULTS}

The first thing we note is the fact that $(\delta S)_{Q, J, M}=0$ on the supersymmetric black hole solutions found in [37]. One might wonder why that is the case, given that (17) has a factor of $T$ in the denominator, and for supersymmetric solutions $T=0$. However, we note that if we take $\tilde{Q}=$ $\tilde{Q}_{\mathrm{BPS}}+\delta Q$ and $\tilde{J}=\tilde{J}_{\mathrm{BPS}}+\delta J$, with $\delta Q, \delta J \ll 1$, we get $T=\mathcal{O}(\delta Q, \delta J)$, whereas $\Delta=\mathcal{O}\left(\delta Q^{2}, \delta Q \delta J, \delta J^{2}\right)$. This means $(\delta S)_{Q, J, M}=\mathcal{O}(\delta Q, \delta J)$ in Eq. (17), i.e., it vanishes in the supersymmetric limit. Another way to see this result is to note that one can read off the change in entropy due to stringy corrections at constant chemical potential $\mu$, temperature $T$, and angular velocity $\Omega$ using the standard thermodynamic relation $S=-(\partial G / \partial T)_{\Omega, \mu}$. In this limit, we get that the correction to the entropy is finite at extremality, being zero in the supersymmetric limit. To our knowledge, there is no a priori reason, based on bulk physics, for why the entropy in the supersymmetic limit is not corrected via stringy effects. This lends support in favor of the index picture advocated in [21-23,25-36].

Second, the sign of $(\delta S)_{Q, J, M}$ appears consistent with the weak gravity conjecture [63], similarly to the analogous calculations in flat space [64-68] and with AdS asymptotics [69]. In particular, one can show using the generalization of the Goon-Penco relation to AdS $[68,69]$ that the leading correction to the extremality bound at fixed energy $M$, charge $Q$, and angular momentum $J$ necessarily decreases with respect to the uncorrected solution. This relation is in perfect agreement with the weak gravity conjecture $[64,66,68]$.

Third, we point out that our final expression (16) only assumes equal angular momenta and equal charges. Notably, it is nonvanishing for a generic nonsupersymmetric extremal black hole and is even valid away from extremality. It would be interesting to understand whether the methods used in [70] could be extended to capture the leading $\alpha^{\prime}$ corrections presented in this paper. Further, this then offers a prediction for the quantum field theoretic calculation. Even though the counting of the supersymmetric states is not corrected at finite $\lambda$, the counting including nonsupersymmetric states should be, and its form should be given by (16). However, as of yet, there are no techniques capable of computing a partition function at strong coupling without the aid of supersymmetry. Though we should mention that in [71] some progress has been reported in going slightly beyond the supersymmetric limit.

Our results rely heavily on [60], since we solely use the uncorrected solution to determine the thermodynamic properties of the corrected solution. In principle, we could use the equations of motion that follow from (12) together with the modified self-duality condition of [42] to 
determine directly the stringy corrected black holes. Under such circumstances, we could determine all thermodynamic properties from the solutions per se instead of using the arguments presented in [60]. Perhaps our current results suggest that the uncorrected supersymmetric solution might be a solution of the corrected equations of motion. This phenomenon has been recently observed in [72] for a number of corrections and black hole solutions. We leave this avenue of research for the future.

Finally, an interesting avenue for future work is to generalize this calculation to the case when all the angular momenta and charges are distinct, using the results from [62]. The complexity of this solution is quite daunting, and computing these corrections would necessarily require more computing power and a more efficient algorithm [73].

\section{ACKNOWLEDGMENTS}

We thank Joonho Kim, Juan Maldacena, Prahar Mitra, and Edward Witten for helpful discussions and Óscar Dias, Joonho Kim, Harvey Reall, Philip Clarke, and Maeve Madigan for comments on a draft of this paper. The work of J. E. S. was supported in part by STFC Grants No. PHY1504541 and No. ST/P000681/1. J.E. S. also acknowledges support from a J. Robert Oppenheimer Visiting Professorship. J.F.M. thanks the Cambridge Trust for his Vice-Chancellors award to support his studies. J. F. M. would particularly like to thank many of his friends and family for enduring the frustration with all the 2075 versions of the code that were off by a minus sign or a factor of 2 .
[1] D. Christodoulou, Reversible and Irreversible Transformations in Black Hole Physics, Phys. Rev. Lett. 25, 1596 (1970).

[2] S. Hawking, Gravitational Radiation from Colliding Black Holes, Phys. Rev. Lett. 26, 1344 (1971).

[3] B. Carter, Rigidity of a black hole, Nat. Phys. Sci. 238, 71 (1972).

[4] J. Bekenstein, Black holes and the second law, Lett. Nuovo Cimento 4, 737 (1972).

[5] D. Christodoulou and R. Ruffini, Reversible transformations of a charged black hole, Phys. Rev. D 4, 3552 (1971).

[6] J. D. Bekenstein, Black holes and entropy, Phys. Rev. D 7, 2333 (1973).

[7] J. M. Bardeen, B. Carter, and S. Hawking, The Four laws of black hole mechanics, Commun. Math. Phys. 31, 161 (1973).

[8] S. Hawking, Black hole explosions, Nature (London) 248, 30 (1974).

[9] S. Hawking, Particle creation by black holes, Commun. Math. Phys. 43, 199 (1975); Erratum, Commun. Math. Phys. 46, 206 (1976).

[10] A. Strominger and C. Vafa, Microscopic origin of the Bekenstein-Hawking entropy, Phys. Lett. B 379, 99 (1996).

[11] J. Breckenridge, D. Lowe, R. C. Myers, A. Peet, A. Strominger, and C. Vafa, Macroscopic and microscopic entropy of near extremal spinning black holes, Phys. Lett. B 381, 423 (1996).

[12] C. G. Callan and J. M. Maldacena, D-brane approach to black hole quantum mechanics, Nucl. Phys. B472, 591 (1996).

[13] G. T. Horowitz and A. Strominger, Counting States of Near Extremal Black Holes, Phys. Rev. Lett. 77, 2368 (1996).

[14] M. Cyrier, M. Guica, D. Mateos, and A. Strominger, Microscopic Entropy of the Black Ring, Phys. Rev. Lett. 94, 191601 (2005).
[15] J. M. Maldacena, The large N limit of superconformal field theories and supergravity, Int. J. Theor. Phys. 38, 1113 (1999).

[16] S. S. Gubser, I. R. Klebanov, and A. M. Polyakov, Gauge theory correlators from noncritical string theory, Phys. Lett. B 428, 105 (1998).

[17] E. Witten, Anti-de Sitter space and holography, Adv. Theor. Math. Phys. 2, 253 (1998).

[18] O. Aharony, S. S. Gubser, J. M. Maldacena, H. Ooguri, and Y. Oz, Large $\mathrm{N}$ field theories, string theory and gravity, Phys. Rep. 323, 183 (2000).

[19] J. Kinney, J. M. Maldacena, S. Minwalla, and S. Raju, An index for 4 dimensional super conformal theories, Commun. Math. Phys. 275, 209 (2007).

[20] S. M. Hosseini, K. Hristov, and A. Zaffaroni, An extremization principle for the entropy of rotating BPS black holes in $\mathrm{AdS}_{5}$, J. High Energy Phys. 07 (2017) 106.

[21] A. Cabo-Bizet, D. Cassani, D. Martelli, and S. Murthy, Microscopic origin of the Bekenstein-Hawking entropy of supersymmetric $\mathrm{AdS}_{5}$ black holes, J. High Energy Phys. 10 (2019) 062.

[22] S. Choi, J. Kim, S. Kim, and J. Nahmgoong, Large AdS black holes from QFT, arXiv:1810.1206.

[23] F. Benini and P. Milan, Black Holes in $4 \mathrm{~d} \mathcal{N}=4$ SuperYang-Mills, Phys. Rev. X 10, 021037 (2020).

[24] M. Honda, Quantum black hole entropy from 4d supersymmetric Cardy formula, Phys. Rev. D 100, 026008 (2019).

[25] A. Arabi Ardehali, Cardy-like asymptotics of the $4 \mathrm{~d} \mathcal{N}=4$ index and $\mathrm{AdS}_{5}$ blackholes, J. High Energy Phys. 06 (2019) 134.

[26] A. Zaffaroni, Lectures on AdS black holes, holography and localization, in CERN Winter School on Strings and Fields 2017 and ICTP School on Supersymmetric Localization, Holography and Related Topics, arXiv:1902.0717.

[27] J. Kim, S. Kim, and J. Song, A 4d $N=1$ Cardy formula, arXiv:1904.0345. 
[28] A. Cabo-Bizet, D. Cassani, D. Martelli, and S. Murthy, The asymptotic growth of states of the $4 \mathrm{~d} \mathcal{N}=1$ superconformal index, J. High Energy Phys. 08 (2019) 120.

[29] A. Gonzlez Lezcano and L. A. Pando Zayas, Microstate counting via Bethe Anstze in the $4 \mathrm{~d} \mathcal{N}=1$ superconformal index, J. High Energy Phys. 03 (2020) 088.

[30] A. Lanir, A. Nedelin, and O. Sela, Black hole entropy function for toric theories via Bethe Ansatz, J. High Energy Phys. 04 (2020) 091.

[31] A. Cabo-Bizet and S. Murthy, Supersymmetric phases of $4 d$ $\mathrm{N}=4$ SYM at large N, arXiv:1909.0959.

[32] A. Cabo-Bizet, D. Cassani, D. Martelli, and S. Murthy, The large- $N$ limit of the $4 \mathrm{~d} \mathcal{N}=1$ superconformal index, arXiv:2005.1065.

[33] S. Murthy, The growth of the $\frac{1}{16}$-BPS index in $4 \mathrm{~d} \mathcal{N}=4$ SYM, arXiv:2005.1084.

[34] P. Agarwal, S. Choi, J. Kim, S. Kim, and J. Nahmgoong, AdS black holes and finite $\mathrm{N}$ indices, arXiv:2005.1124.

[35] F. Benini, E. Colombo, S. Soltani, A. Zaffaroni, and Z. Zhang, Superconformal indices at large $N$ and the entropy of $\mathrm{AdS}_{5} \times \mathrm{SE}_{5}$ black holes, arXiv:2005.1230.

[36] A. Gadde, Lectures on the superconformal index, arXiv:2006.1363.

[37] J. B. Gutowski and H. S. Reall, Supersymmetric AdS(5) black holes, J. High Energy Phys. 02 (2004) 006.

[38] J. B. Gutowski and H. S. Reall, General supersymmetric AdS(5) black holes, J. High Energy Phys. 04 (2004) 048.

[39] H. K. Kunduri, J. Lucietti, and H. S. Reall, Supersymmetric multi-charge $\operatorname{AdS}(5)$ black holes, J. High Energy Phys. 04 (2006) 036.

[40] B. Bates and F. Denef, Exact solutions for supersymmetric stationary black hole composites, J. High Energy Phys. 11 (2011) 127.

[41] A. Dabholkar, S. Murthy, and D. Zagier, Quantum black holes, wall crossing, and mock modular forms, arXiv:1208.4074.

[42] M.F. Paulos, Higher derivative terms including the Ramond-Ramond five-form, J. High Energy Phys. 10 (2008) 047.

[43] We would like to note, however, that Ref. [42] has a number of typos in their Sec. IV, which summarizes their results.

[44] M. B. Green and C. Stahn, D3-branes on the Coulomb branch and instantons, J. High Energy Phys. 09 (2003) 052.

[45] M. Cvetic, H. Lu, and C. Pope, Charged Kerr-de Sitter black holes in five dimensions, Phys. Lett. B 598, 273 (2004).

[46] J. York and W. James, Role of Conformal Three Geometry in the Dynamics of Gravitation, Phys. Rev. Lett. 28, 1082 (1972).

[47] G. Gibbons and S. Hawking, Action integrals and partition functions in quantum gravity, Phys. Rev. D 15, 2752 (1977).

[48] V. Balasubramanian and P. Kraus, A stress tensor for anti-de Sitter gravity, Commun. Math. Phys. 208, 413 (1999).

[49] S. de Haro, S. N. Solodukhin, and K. Skenderis, Holographic reconstruction of space-time and renormalization in the AdS/CFT correspondence, Commun. Math. Phys. 217, 595 (2001).
[50] To avoid cluttering in the notation, from here onward we take $Q \geq 0$ and $J \geq 0$.

[51] G. J. Loges, T. Noumi, and G. Shiu, Duality and supersymmetry constraints on the weak gravity conjecture, arXiv:2006.0669.

[52] J. Markeviciute and J.E. Santos, Hairy black holes in $\mathrm{AdS}_{5} \times \mathrm{S}^{5}$, J. High Energy Phys. 06 (2016) 096.

[53] J. Markeviciute, Rotating hairy black holes in $\mathrm{AdS}_{5} \times \mathrm{S}^{5}$, J. High Energy Phys. 03 (2019) 110.

[54] These is not the only restrictions on the three-dimensional Moduli space of black hole solutions $\left\{\tilde{J}, \tilde{Q}, r_{+}\right\}$that bulk regularity demands, but it is the only one we will need to show that $\delta S>0$.

[55] A. Chamblin, R. Emparan, C. V. Johnson, and R. C. Myers, Charged AdS black holes and catastrophic holography, Phys. Rev. D 60, 064018 (1999).

[56] M. Cvetic, M. Duff, P. Hoxha, J. T. Liu, H. Lu, J. Lu, R. Martinez-Acosta, C. Pope, H. Sati, and T. A. Tran, Embedding AdS black holes in ten-dimensions and eleven-dimensions, Nucl. Phys. B558, 96 (1999).

[57] M. Cvetic, H. Lu, C. Pope, A. Sadrzadeh, and T. A. Tran, Consistent $\mathrm{SO}(6)$ reduction of type IIB supergravity on S5, Nucl. Phys. B586, 275 (2000).

[58] As usual, we use this term with a certain abuse of notation, because the five-form $F_{(5)}$ is only made self-dual at the level of the equations of motion. After the inclusion of the correction term proportional to $\gamma$, the self-duality condition is accordingly changed.

[59] Note that after computing $\mathcal{T}$ with this expression, one still needs to antisymmetrize over the first three indices and the last three indices and then symmetrize for their exchange, before plugging into the monomials.

[60] H. S. Reall and J. E. Santos, Higher derivative corrections to Kerr black hole thermodynamics, J. High Energy Phys. 04 (2019) 021.

[61] See Supplemental Material at http://link.aps.org/ supplemental/10.1103/PhysRevD.103.066008 for details on the implementation and consistency checks on evaluating the $\alpha^{\prime}$ corrections.

[62] S.-Q. Wu, General nonextremal rotating charged AdS black holes in five-dimensional $U(1)^{3}$ gauged supergravity: A simple construction method, Phys. Lett. B 707, 286 (2012).

[63] N. Arkani-Hamed, L. Motl, A. Nicolis, and C. Vafa, The string landscape, black holes and gravity as the weakest force, J. High Energy Phys. 06 (2007) 060.

[64] Y. Kats, L. Motl, and M. Padi, Higher-order corrections to mass-charge relation of extremal black holes, J. High Energy Phys. 12 (2007) 068.

[65] Y. Hamada, T. Noumi, and G. Shiu, Weak Gravity Conjecture from Unitarity and Causality, Phys. Rev. Lett. 123, 051601 (2019).

[66] C. Cheung, J. Liu, and G. N. Remmen, Proof of the weak gravity conjecture from black hole entropy, J. High Energy Phys. 10 (2018) 004.

[67] G. J. Loges, T. Noumi, and G. Shiu, Thermodynamics of 4D dilatonic black holes and the weak gravity conjecture, Phys. Rev. D 102, 046010 (2020).

[68] G. Goon and R. Penco, Universal Relation Between Corrections to Entropy and Extremality, Phys. Rev. Lett. 124, 101103 (2020). 
[69] S. Cremonini, C. R. Jones, J. T. Liu, and B. McPeak, Higher-derivative corrections to entropy and the weak gravity conjecture in anti-de Sitter space, arXiv:1912.1116.

[70] M. David, J. Nian, and L. A. Pando Zayas, Gravitational Cardy limit and AdS black hole entropy, arXiv:2005.1025.

[71] F. Larsen, J. Nian, and Y. Zeng, $\mathrm{AdS}_{5}$ black hole entropy near the BPS limit, J. High Energy Phys. 06 (2020) 001.
[72] N. Bobev, A. M. Charles, K. Hristov, and V. Reys, The unreasonable effectiveness of higher-derivative supergravity in $\mathrm{AdS}_{4}$ holography, arXiv:2006.0939.

[73] For the interested reader, even just checking that the solution [62] indeed solves the equations of motion as claimed takes a few hours with a rather optimized Mathematica code. 